\title{
Biological and Therapeutic Effects of Troxerutin: Molecular Signaling Pathways Come into View
}

\author{
Zahra Ahmadi ${ }^{1}$, Reza Mohammadinejad ${ }^{2}$, Sahar Roomiani $^{1}$, Elham Ghasemipour Afshar ${ }^{3}$, \\ Milad Ashrafizadeh ${ }^{4}$ * \\ ${ }^{1}$ Department of Basic Science, Faculty of Veterinary Medicine, Islamic Azad Branch, University of Shushtar, Khuzestan, Iran \\ ${ }^{2}$ Pharmaceutics Research Center, Institute of Neuropharmacology, Kerman University of Medical Sciences, Kerman, Iran \\ ${ }^{3}$ Department of Microbiology, Faculty of Science, Islamic Azad University, Kerman, Iran \\ ${ }^{4}$ Department of Basic Science, Faculty of Veterinary Medicine, University of Tabriz, Tabriz, Iran
}

Received April 13, 2019

Reviewed April 19, 2019

Accepted March 4, 2021

* Corresponding Author

Milad Ashrafizadeh

Department of Basic Science, Faculty of

Veterinary Medicine, University of Tabriz,

Tabriz, Iran

Tel: +98-903-236-0639

E-mail: dvm.milad73@yahoo.com
Flavonoids consist a wide range of naturally occurring compounds which are exclusively found in different fruits and vegetables. These medicinal herbs have a number of favourable biological and therapeutic activities such as antioxidant, neuroprotective, renoprotective, anti-inflammatory, anti-diabetic and anti-tumor. Troxerutin, also known as vitamin P4, is a naturally occurring flavonoid which is isolated from tea, coffee and cereal grains as well as vegetables. It has a variety of valuable pharmacological and therapeutic activities including antioxidant, anti-inflammatory, anti-diabetic and anti-tumor. These pharmacological impacts have been demonstrated in in vitro and in vivo studies. Also, clinical trials have revealed the efficacy of troxerutin for management of phlebocholosis and hemorrhoidal diseases. In the present review, we focus on the therapeutic effects and biological activities of troxerutin as well as its molecular signaling pathways.

Keywords: troxerutin, flavonoid, herbal medicine, biological activity, therapeutic effect

\section{ABBREVIATIONS}

PCNA, proliferating cell nuclear antigen; $\mathrm{CP}$, cisplatin; OTA, ochratoxin; SOD, superoxide dismutase; BDE-47, 2,2'-4,4'-tetrabromodiphenyl ether; ACR, albumin-to-creatinine ratio; OX2, cyclooxygenase-2; GR, glutamate reductase; iNOS, inducible nitric oxide synthase; G6PD, glucose-6-phosphate dehydrogenase; NF-kB, nuclear factor kappa-light-chain-enhancer of activated B cells; CXCR4, C-X-C chemokine ligand 12 recptor 4; HO-1, heme oxygenase-1; TXNIP, thioredoxin interaction protein; Bcl-2, b-cell lymphoma 2; Ni, nickel; ROS, reactive oxygen species; CMS, chronic mild stress; CFTR, cystic fibroblast transmembrane conductance regulator; CLC2, chloride channel 2; NLRP3, nod-like receptor subset inflammasome; HFD, high-fat diet; NOX2, NADPH oxidase 2; Nrf2, nuclear factor erythroid 2-related factor-2; Ab, amyloid beta; PARP, poly ADP ribose polymerase; BBB, blood-brain barrier; L-NAME, N-nitro-L-arginine methyl ester hydrochloride; TBARS, thiobarbituric acid reactive substances; GSH, glutathione; $\mathrm{AChE}$, acetylcholinesterase; CAT, catalase; STAT3, signal transducers and activation of transcription 3; 2-AA, 2-aminoantherace; TH, tyrosinehydroxylase; TCHI, troxerutin and cerebroprotein hydrosylate; eNOS, endothelial nitric oxide synthase; NO, nitric oxide; ER $\beta$, esterogen receptor $\beta$; PI3K, phosphatidylinositol 3-kinase; 6-OHDA, 6-hydroxydopamine; GCLC, glutamate cysteine ligase catalytic; GCLM, glutamate cysteine ligase modifier; MDA, malondialdehyde; GFAP, glial fibrillary acid protein; AMPK, adenosine monophosphate-activated protein kinase; SIRT1, silent mating type information regulation 2 homolog 1; TID, type I diabetes; GPX, glutathione peroxide; TNF- $\alpha$, tumor necrosis factor- $\alpha$; IRS1, insulin receptor substrate 1 ; INF- $\gamma$, interferon- $\gamma$; CK, creatinine kinase; TAC, total antioxidant capacity; IRAK1, interleukin-1 receptor-associated kinase 1; TRAF6, transmembrane factor receptor-associated factor 6; I/R, ischemic/reperfusion; PCV, premature ventricular; rBMECs, rat brain microvascular endothelial cells; AST, aspartate aminotransferase; NOD, nu- 
cleotide oligomerization domain; $\mathrm{LDH}$, lactate dehydrogenase; ICAM-1, intercellular adhesion molecule-1; HFFD, high fat or fructose diet; RIP2, interacting protein-2; cTnI, cardiac troponin I; ER, endoplasmic reticulum.

\section{INTRODUCTION}

From the beginning of drug discovery revolution, a large number of naturally occurring compounds have been discovered and over the past decades, much attention has been made towards the medicinal herbs which is due to their low side effects and valuable biological activities. The dietary intake of vegetables and fruits has demonstrated to have health promoting effects which are because of the presence of bioactive compounds having a number of pharmacological effects such as anti-oxidant, anti-inflammatory, anti-tumor, anti-diabetic, antiobesity and so on $[1,2]$. Dietary flavonoids comprise a vase range of polyphenolic compounds exclusively found in fruits, vegetables, grains, herbs and beverages [3]. Flavonoids share a common benzo- $\gamma$-pyrone structure which are synthesized in response to microbial infection through phenylpropanoid route [4]. It has been reported that the consumption of flavonoids is at the range of 20 to $1,000 \mathrm{mg} /$ day and their bioavailability is various among individuals. Flavonoids are absorbed either by small or large intestines and a large part of them is exposed to the colonic microbiota. Then, the microbiome digests flavonoids into phenolic and aromatic acids to facilitate their absorption $[5,6]$.

Troxerutin, also known as vitamin $\mathrm{P}_{4}$, is a derivative of naturally occurring bioflavonoid rutin and is extensively found in tea, coffee, cereal grains and a number of vegetables and fruits. It has been shown that troxerutin can be significantly absorbed by the gastrointestinal system because of its high water solubility and exerts protective effects without making cytotoxic impact. This naturally occurring compound has various favorable biological activities such as antioxidant, anti-inflammatory, anti-diabetic and anti-tumor. Besides, clinical trials have demonstrated the efficacy of troxerutin in management of phlebocholosis and hemorrhoidal diseases which is associated with capability of troxerutin in promoting microcirculation and protection of endothelial cells [7-15]. In this review, we present a mechanistic introduction about the pharmacological effects of troxerutin (Table 1).

\section{PHARMACOLOGICAL EFFECTS}

\section{Renoprotective effect}

Kidney is one of the important organs for secretion, reabsorption of aminoacids and glucose, production of active form of vitamin $\mathrm{D}$, and also plays a significant role in immunologic and metabolic functions [16-20]. Troxerutin has shown high efficacy in protection of kidney (Fig. 1). In a study, the protective effect of troxerutin in gentamycin-induced acute kidney injury was examined [21]. The results of this study demonstrated the great protective effect of troxerutin, so that troxerutin administration promoted renal function through enhancing glomerular filtration rate and reducing the levels of urinary albumin, urinary albumin to creatinine ratio, serum creatinine and blood urea nitrogen. Also, troxerutin remarkably alleviated gentamycin-induced renal tissue injury by decreasing expression level of renal tubular injury marker KIM-1, renal histopathological alterations and oxidative stress. Besides, troxerutin administration was associated with decreased level of inflammatory cytokines such as IL-10, TNF- $\alpha$ and IL- 6 and also diminished the level of apoptosis as well as increased the level of tissue regenerative capacity through upregulation of proliferating cell nuclear antigen (PCNA), showing the potential renoprotective effect of troxerutin. Yang and coworkers investigated the protective effect of troxerutin on ochratoxin (OTA)-mediated renal lipotoxicity [22]. The results of this study exhibited the potential effect of troxerutin in renal protection, so that troxerutin administration remarkably attenuated nephrotoxicity, promoted endurance and healed systemic energy metabolism and renal inflammation in OTA-induced nephrotic mice. Also, it was shown that troxerutin is able to stimulate reduction in the levels of triglycerides, phosphatidylcholines and phosphatidylethanolamines in nephropathy. Examination of molecular mechanisms revealed that the effect of troxerutin in decreasing renal injury is associated with enhanced expression of sphingomyelinase, the cystic fibrosis transmembrane conductance regulator and chloride channel 2. Shan and colleagues investigated the protective effect of troxerutin against 2.2'-4.4'-tetrabromodiphenyl ether (BDE-47)-induced inflammatory damage in kidney [23]. The results of this study demonstrated that troxerutin significantly decreases ROS level and urine albumin-to-creatinine ration (ACR) and reduces the activities of inflammatory factors such as cyclooxygenase-2 (OX-2), inducible nitric oxide synthase (iNOS) and nuclear factor kappa-light-chain-enhancer of 
Table 1. The valuable biological activities of troxerutin

\begin{tabular}{|c|c|c|c|}
\hline In vitro & In vivo & Major outcomes & Reference \\
\hline- & Diabetic rats & $\begin{array}{l}\text { Increasing the level of testosterone and GPX and improving sperm quality } \\
\text { as well as decreasing blood sugar }\end{array}$ & [67] \\
\hline Brain tissues & - & $\begin{array}{l}\text { Exerting neuroprotective effect through decreasing oxidative stress and } \\
\text { angiogenesis improving }\end{array}$ & {$[16]$} \\
\hline- & Wistar rats & $\begin{array}{l}\text { Exerting renoprotective effect by decreasing oxidative stress, inflammation } \\
\text { and improving glomerular filtration rate }\end{array}$ & [21] \\
\hline- & Albino Wistar rats & $\begin{array}{l}\text { Decreasing oxidative stress and enhancing the level of enzymatic and non- } \\
\text { enzymatic antioxidants }\end{array}$ & [34] \\
\hline Cardiomyocyte & Rat & Exerting anti-apoptotic effect in I/R injury through inhibition of miR-146a-5p & {$[71]$} \\
\hline $\begin{array}{l}\text { Hepatocellular } \\
\text { carcinoma cell line } \\
\text { (HuH-7) }\end{array}$ & - & $\begin{array}{l}\text { Exerting anti-tumor effect by reduction of Nrf2 and } \mathrm{HO}-1 \text { and inhibition of } \\
\text { NF-kB }\end{array}$ & [48] \\
\hline $\begin{array}{l}\text { Hepatocellular } \\
\text { carcinoma cells }\end{array}$ & - & Exerting anti-tumor activity through disruption of MDM2-p53 interaction & [49] \\
\hline- & Mice & Decreasing renal injury through inflammation reduction & {$[22]$} \\
\hline- & Adult male rats & $\begin{array}{l}\text { Decreasing anxiety- and depressive-like behaviors induced by chronic mild } \\
\text { stress }\end{array}$ & {$[55]$} \\
\hline- & Wistar rats & $\begin{array}{l}\text { Improving HFD-induced spatial memory impairments of the offspring via } \\
\text { serum and hippocampal apelin level modulation }\end{array}$ & {$[56]$} \\
\hline- & Wistar rats & $\begin{array}{l}\text { Improving Johns score's, sperm count and levels of FSH and LH and eerting } \\
\text { anti-apoptotic effect in rats exposed to I/R }\end{array}$ & {$[72]$} \\
\hline- & Male rats & $\begin{array}{l}\text { Exerting neuroprotective effect through modulation of endothelial nitric } \\
\text { oxide synthase (eNOS) coupling/decoupling status }\end{array}$ & [57] \\
\hline $\begin{array}{l}\text { Neurons, astrocytes and } \\
\text { rBMECs }\end{array}$ & - & $\begin{array}{l}\text { Exerting protective effect against cerebral I/R injury through decreasing } \\
\text { inflammatory cytokines and proapoptotic factors and upregulation of GAP- } \\
43 \text {, Claudin- } 5 \text { and AQP-4 }\end{array}$ & [73] \\
\hline- & Mice & $\begin{array}{l}\text { Exerting renoprotective effect through CXCR4-TXNIP/NLRP3 signaling } \\
\text { pathway }\end{array}$ & [23] \\
\hline- & Rat & $\begin{array}{l}\text { Improving learning and memory levels and enhancing antioxidant capacity } \\
\text { via Nrf2 upregulation }\end{array}$ & [68] \\
\hline- & Rat & $\begin{array}{l}\text { Decreasing lipid peroxidation markers and increasing nonenzymatic and } \\
\text { enzymatic antioxidants }\end{array}$ & [24] \\
\hline- & Mice & Exerting renoprotective effect & [25] \\
\hline- & Male Wistar rats & $\begin{array}{l}\text { Decreasing inflammatory cytokines and exerting anti-arrhythmogenic effect } \\
\text { in I/R injury of diabetic myocardium }\end{array}$ & [74] \\
\hline- & Female Wistar rat & $\begin{array}{l}\text { Improving anxiety- and depressive-like behaviors in the offspring of HFD diet } \\
\text { dams }\end{array}$ & {$[58]$} \\
\hline- & $\begin{array}{l}\text { Animal model of } \\
\text { Alzheimer's disease }\end{array}$ & $\begin{array}{l}\text { Exerting neuroprotective effect through increasing the activities of SOD and } \\
\text { GPx and decreasing the level of MDA and AChE activity }\end{array}$ & [59] \\
\hline- & $\begin{array}{l}\text { Rat model of myocardial } \\
\text { I/R injury }\end{array}$ & Exerting protective effect against myocardial I/R injury via Akt/PI3K pathway & [75] \\
\hline- & Male mice & $\begin{array}{l}\text { Decreasing ROS production and inhibition of apoptosis through Nrf2 } \\
\text { activation and NOX2 inhibition }\end{array}$ & {$[26]$} \\
\hline- & Mice & Decreasing mitochondrial oxidative stress and myocardial apoptosis & [79] \\
\hline $\begin{array}{l}\text { Hepatocellular } \\
\text { carcinoma cell }\end{array}$ & Cancer bearing animal & $\begin{array}{l}\text { Exerting anti-tumor effect through stimulation of ROS production and } \\
\text { improving liver markers }\end{array}$ & {$[50]$} \\
\hline
\end{tabular}


Zahra Ahmadi, et al.

Table 1. Continued

\begin{tabular}{|c|c|c|c|}
\hline In vitro & In vivo & Major outcomes & Reference \\
\hline $\begin{array}{l}\text { Human gastric cancer } \\
\text { cells }\end{array}$ & Mice xenograft models & $\begin{array}{l}\text { Sensitizing human gastric cancer cells via inhibition of STAT3/NF-kB and } \\
\text { Bcl-2 pathways }\end{array}$ & [51] \\
\hline - & $\begin{array}{l}\text { Rat model of Parkinson's } \\
\text { disease }\end{array}$ & Exerting neuroprotective effect through PI3k/ER $\beta$ induction & {$[60]$} \\
\hline $\begin{array}{l}\text { 3T3-L1 and MDA- } \\
\text { MB-231 cells }\end{array}$ & - & $\begin{array}{l}\text { Inhibition of DNA and 2-aminoanthracene interaction and exerting anti- } \\
\text { mutagenic effect }\end{array}$ & [52] \\
\hline- & Diabetic rats & $\begin{array}{l}\text { Decreasing hippocampal mRNA levels of NF-kB, IRAK-1 and TRAF- } 6 \text { through } \\
\text { miR-146a and miR-155 upregulation }\end{array}$ & [70] \\
\hline Thymocytes & - & $\begin{array}{l}\text { Inhibition of apoptosis induction induced by irradiation through suppressing } \\
\text { PTEN and JNK and induction of Akt }\end{array}$ & [78] \\
\hline- & Rat model of I/R injury & Suppressing cell-cell interaction and release of inflammatory mediators & [76] \\
\hline
\end{tabular}

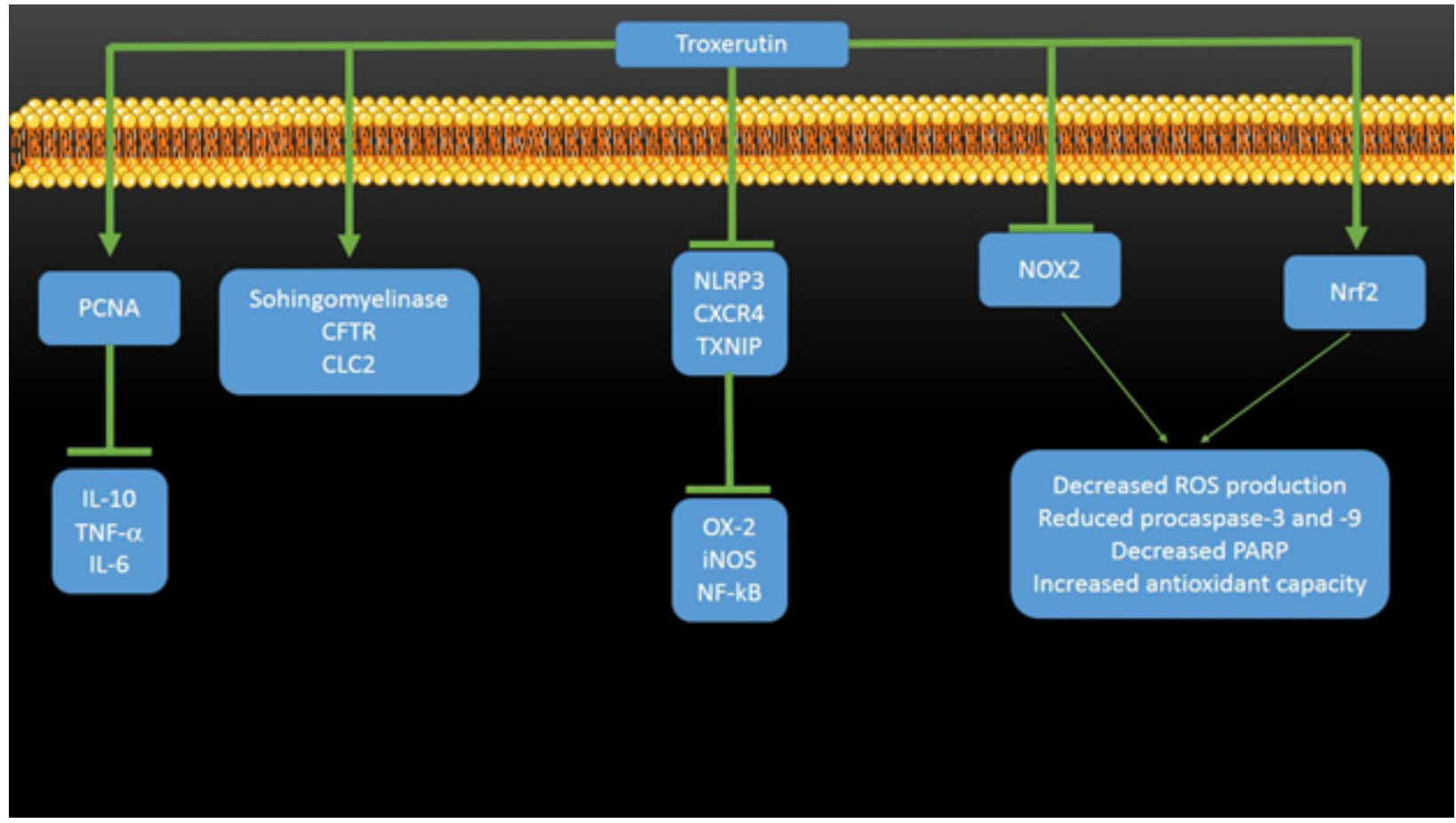

Figure 1. The involvement signaling pathways in modulation of renoprotective effects of troxerutin. PCNA, proliferating cell nuclear antigen; CFTR, cystic fibroblast transmembrane conductance regulator; CLC2, Chloride channel 2; NLRP3, Nod-like receptor subset inflammasome; CXCR4, C-X-C chemokine ligand 12 receptor 4; TXNIP, thioredoxin interaction protein; OX-2, cyclooxygenase-2; iNOS, inducible nitric oxide synthase; NOX2, NADPH oxidase 2; Nrf2, nuclear factor erythroid 2-related factor-2; ROS, reactive oxygen species; PARP, poly ADP ribose polymerase; NF-kB, nuclear factor kappa-light-chain-enhancer of activated B cells. 
activated B cells (NF-kB) in kidney tissues exposed to BDE-47. Also, troxerutin administration decreased the expression level of NLRP3 inflammasome and C-X-C chemokine ligand 12 receptor 4 (CXCR4) as well as thioredoxin interaction protein (TXNIP), showing the role of CXCR4-TXNIP/NLRP3 pathway in renoprotective effect of troxerutin. Elangovan and colleagues have indicated the protective effect of troxerutin on nickle (Ni)-induced renal dysfunction [24]. Biochemically, troxerutin decreased the levels of lipid peroxidation merkers, while it enhanced the levels of nonenzymatic and enzymatic antioxidants. Histopathologically, troxerutin maintained normal histologic structure of renal tissue, demonstrating the nephroprotective potential of troxerutin. In another study, Dehnamaki and coworkers evaluated the effect of troxerutin on the cisplatin (CP)induced kidney injury in mice [25]. It was found that troxerutin administration decreases serum creatinine, blood urea nitrogen and MDA levels, enhances the activities of SOD and glutathione peroxidase, and improves histopathological alterations of kidney tissue. In a study, the renoprotective effect of troxerutin against BDE-47-mediated kidney damage was evaluated [26]. It was shown that troxerutin decrease renal cell apoptosis and urinary protein secretion and suppresses the release of cytochrome $\mathrm{C}$ from mitochondria with increasing Bcl-2/Bax ratio. Besides, troxerutin prevents the activation of procaspase- 9 and procaspase- 3 and the cleavage of PARP, decrease the production of ROS and enhances the antioxidant activity. It was found that these protective effects are mediated by inhibition of NOX2 activity and stimulation of Nrf2 activity.

\section{Antioxidant effect}

Oxidative stress is defined as a condition which the balance of antioxidant defense system is impaired, and proteins, lipids and DNA expose to excess oxidative molecules, resulting in oxidation of lipids and proteins and DNA damage. The studies show the potential role of oxidative stress in pathological conditions [27-33]. So, a number of drugs has been designed with this strategy. Troxerutin has shown high antioxidant activity. In a study, the effect of troxerutin on the N-nitro-L-arginine methyl ester hydrochloride (L-NAME)-mediated oxidative stress was examined [34]. It was found that troxerutin is able to reverse all the harmful effects of L-NAME, so that troxerutin administration decreased the level of thiobarbituric acid reactive substances (TBARS) and effectively enhanced the activities of enzymatic and non-enzymatic antioxidants such as glutathione (GSH), vitamin $\mathrm{C}$ and vitamin $\mathrm{E}$. In a study, the antioxidant activity of troxerutin and its acylated derivatives was investigated [35]. The results of hydroxyl radical, ABTS and ORAC assays demonstrated that troxerutin and its acylated derivatives have similar antioxidant activity, whereas acylated derivatives of troxerutin exhibited higher inhibitory effect on 2,2'-azobis (2-amidinopropane) dihydrochloride-induced erythrocyte hemolysis compared to troxerutin. In another study, the ameliorative effect of troxerutin on the nickel $(\mathrm{Ni})$-induced testicular toxicity in Wistar rats was examined [36]. The administration of troxerutin effectively improved SOD, catalase (CAT), GPx, GST, glutathione reductase (GR), glucose-6-phosphate dehydrogenase (G6PD), glutathione, ascorbate, total sulfhydryl groups and testis-organ weight, whereas it significantly decreased the accumulation of $\mathrm{Ni}$, lipid peroxidation products and protein carbonyl levels. Panat and colleagues examined the protective effect of troxerutin against oxidative stress-induced cell death [37]. The results of this study demonstrated that troxerutin protects a number of cells such as epithelial cells, fibroblasts and lymphocytes against peroxyl radical-induced apoptosis, necrosis and mitotic death through scavenging intracellular basal and inducible ROS levels and enhancing intracellular GSH levels.

\section{Anti-tumor effect}

In spite of progress and advancement in cancer prevention and treatment, it is still one of the most challenge diseases around the world [38-47]. Troxerutin has indicated great antitumor activity and a number of studies showing its anti-tumor effect are included in this review (Fig. 2). In a study, the antitumor activity of troxerutin against hepatocellular carcinoma cell line was assessed [48]. The results of this study demonstrated that troxerutin exerts anti-tumor activity on $\mathrm{HuH}-7$ cells by induction of heme oxygenase ( $\mathrm{HO})-1$ and nuclear translocation of Nrf2. Also, it was shown that troxerutin reduces the nuclear translocation of NF-kB (p65 subunit) through LKKB downregulation and consequently, resulting in decreased inflammatory responses, proliferation and cell survival. In another study, the inhibitory effect of troxerutin on hepatic tumorigenesis was evaluated [49]. It was shown that troxerutin diminishes hepatic nodule formation, changes in enzymatic status, frequencies of glutathione-s-transferase and PCNA and marker of S phase progression. Besides, it was found that troxerutin administration is associated with impairment in MDMd-p53 interaction, resulting in mitoinhibitory effect of troxerutin in hepatic tu- 


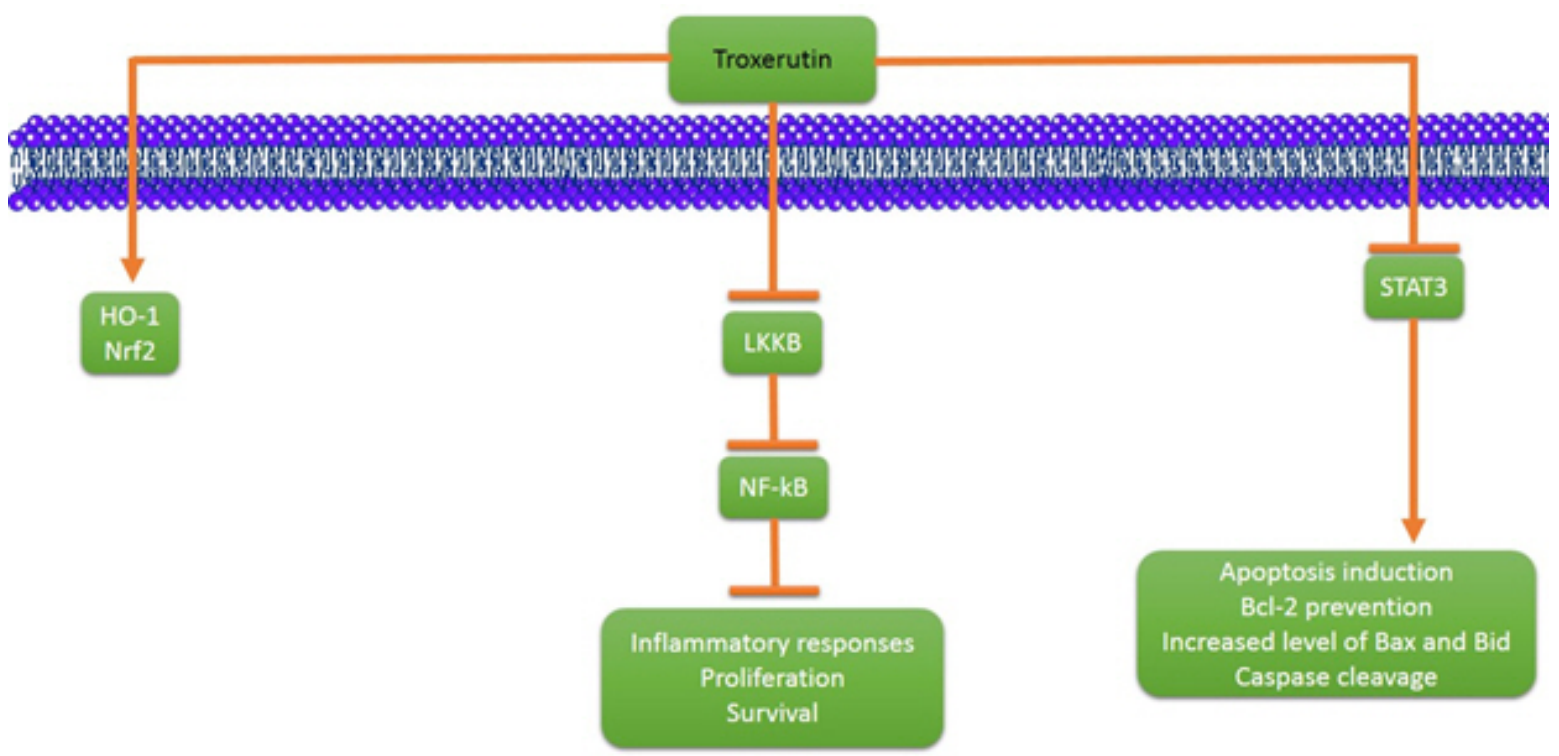

Figure 2. The involvement signaling pathways in stimulation of anti-tumor activity of troxerutin. H0-1, heme oxygenase-1; Nrf2, nuclear factor erythroid 2-related factor-2; NF-kB, nuclear factor kappa-light-chain-enhancer of activated B cells; STAT3, signal transducer and activation of transcription 3; Bcl-2, b-cell lymphoma 2.

morigenesis. In a study, the anti-tumor activity of troxerutin against hepatocellular carcinoma was examined [50]. In vitro experiment demonstratd that troxerutin exerts anti-tumor activity and decreases the viability of HuH-7 cells through ROS production. Also, in vivo experiment indicated that troxerutin is able to reduce the survival of cancer cells through promoting liver markers and decreasing GST-P, 8-OHdG and Ki-67 expression. In another study, $\mathrm{Xu}$ and Jang evaluated the effect of troxerutin on the efficacy of 5-fluorouracil (5-Fu) in treatment of human gastric cancer [51]. It was found that a combination of troxerutin and 5-Fu exerts inhibitory effect on the growth of human gastric cancer cells and results in inhibition of cell proliferation in a dose-dependent manner. It was shown that troxerutin suppresses $\mathrm{p} 65$ by downregulating signal transducers and activation of transcription 3 (STAT3) and sensitizes cancer cells to 5 -FuU apoptosis through Bcl-2 prevention. Furthermore, the pro-apoptotic proteins such as Bax and Bid were increased and caspase was cleaved, resulting in apoptosis induction, demonstrating the potential of troxerutin in treatment of human gastric cancer. Subastri and coworkers investigated the effect of troxerutin on DNA and 2-aminoantheracene (2-AA) interaction [52]. Fluorescence spectroscopy showed the direct contact of 2-AA and DNA which was prevented by troxerutin. Also, troxerutin administration was associated with inhibition of 2-AA and UVA radioation-mediated damage. Anti-mutagen- icity study demonstrated that troxerutin suppresses mutation induced colony formation in mutant strain of S. typhymurium. Thomas and colleagues examined antineoplastic impact of troxerutin on rat preneoplastic liver [53]. Troxerutin administration improved enzyme activities and hepatic structure in the hepatocellular carcinoma-bearing rat. Also, troxerutin remarkably suppressed DNA damage, cell proliferation, inflammation, fibrosis and hepatic hyperplasia. In another study, Panat and coworkers introduced a novel method of function of troxerutin [54]. The redioresistant (DU145) and sensitive (PC3) prostate cancer were used, and it was shown that pretreatment with troxerutin and $\gamma$-radiation enhances cytotoxicity through ROS production and DNA damage. It was found that DNA binding at minor groove by troxerutin leads to the strand breaks and subsequently, elevated radiation induced cell death.

\section{Neuroprotective effect}

A number of studies have evaluated the neuroprotective effect of troxerutin which are included in this review (Fig. 3). In a study, the protective effect of troxerutin and cerebroprotein hydrosylate (TCHI) on the cerebral ischemia was investigated [16]. It was shown that this combination increases the lactate dehydrogenase levels and SOD activity, whereas it decreases lactic acid and MDA levels in ischemic brain tissues. Besides, 


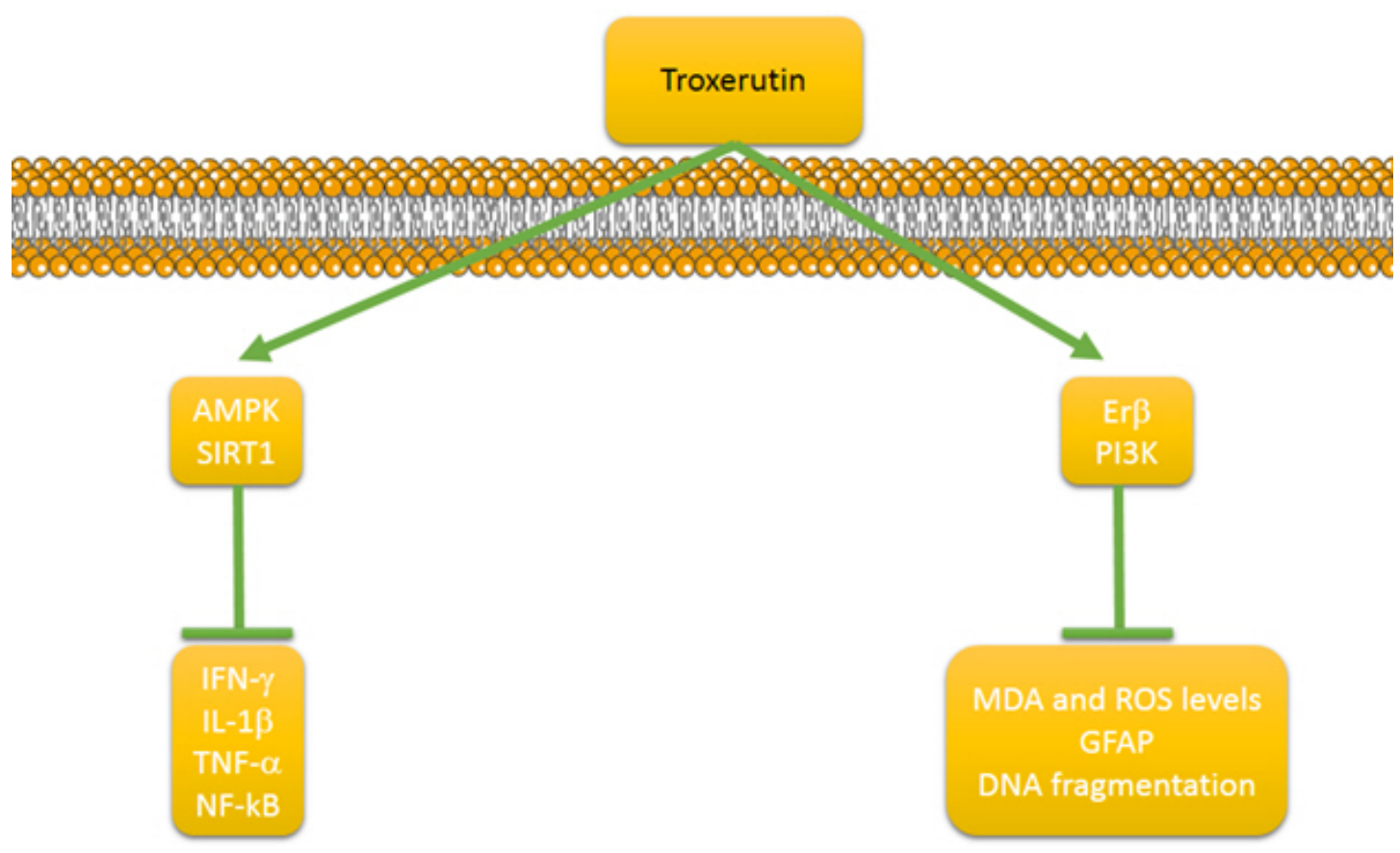

Figure 3. The involvement signaling pathways in neuroprotective effect of troxerutin. PI3K, phosphatidylinositol 3-kinase; ER $\beta$, esterogen receptor $\beta$; MDA, malondialdehyde; ROS, reactive oxygen species; GFAP, glial fibrillary acid protein; AMPK, adenosine monophosphate-activated protein kinase; SIRT1, silent mating type information regulation 2 homolog 1; TNF- $\alpha$, tumor necrosis factor $\alpha$; INF- $\gamma$, interferon- $\gamma$.

TCHI induced microvessel formation and enhanced vascular maturation mechanisms such as proliferation, adhesion, migration and tube formation. Azarfarin and colleagues investigated the effect of troxerutin on the anxiety- and depressive-like behaviors mediated by chronic mild stress (CMS) [55]. The results of this study demonstrated that troxerutin effectively diminishes immobility and enhances swimming time. Also, troxerutin elevates open arm entrance and time spent in the center and number of entrances crossing, while it decreases serum cortisol levels, demonstrating the efficacy of troxerutin in protection against CMS. In a study, the protective effect of troxerutin on maternal high-fat diet (HFD)-induced impairments of spatial memory and apelin in male offspring was assessed [56]. The results of this study revealed that troxerutin administration remarkably enhances traveled distance and time spent, and also serum and hippocampal levels of HFDfed dams, whereas it diminishes the serum apelin levels in damsm showing the potential of troxerutin in improving HFDinduced spatial memory impairments of the offspring. Zhao and coworkers examined the protective effect of TCHI against damage induced by traumatic brain injury (TBI) [57]. It was shown that TCHI leads to attenuation of neurological disorders, decrease of infarct volume, promotion of proliferation, endothelial cell loss and blood-brain barrier (BBB) integrity. It was found that these neuroprotective effects of troxerutin are mediated by modulation of endothelial nitric oxide synthase (eNOS) coupling/decoupling status that not only enhanced nitric oxide (NO), but also diminished peroxynitrate level expression. Bayandor and colleagues examined the potential effect of troxerutin on anxiety- and depressive-like behaviors in the offspring of HFD fed dams [58]. HFD offspring demonstrated higher anxiety and depressive-like behaviors, higher blood sugar, cholesterol and cortisol levels compared to the control groups. In a study, the protective effect of troxerutin against the amyloid beta $(A \beta)$-induced oxidative stress and apoptosis was investigated [59]. It was shown that troxerutin administration significantly alleviates MDA levels and acetylcholinesterase (AChE) activity and enhances SOD and GPx activities in the hippocampus. Furthermore, troxerutin administration was related to the decreased number of apoptotic cells. In a study, the involvement of phosphatidylinositol 3-kinase (PI3K)/estrogen receptor $\beta$ (ER $\beta$ ) signaling pathway in neuroprotective effect of troxerutin in 6-hydroxydopamine (6-OHDA) lesion rat model of Parkinson's disease was evaluated [60]. It was shown that troxerutin decreases apomorphine-induced motor asymmetry and reduces striatal MDA and ROS levels. Also, troxerutin 
administration diminished glial fibrillary acid protein (GFAP) as a marker of astrogliosis, and DNA fragmentation as well as inhibited the loss of nigral tyrosine hydroxylase $(\mathrm{TH})$-positive neurons. It was found that these neuroprotective effects of troxerutin are mediated by stimulation of ER $\beta$ and PI3K signaling pathways. Zhang and coworkers evaluated the neuroprotective effect of troxerutin in T1DM rats [61]. Biochemically, troxerutin administration decreased escape latency and MDA levels, whereas it enhanced glutamate cysteine ligase modifier (GCLM) and glutamate cysteine ligase catalytic (GCLC) levels as well as SOD activity and GSH levels. Histopathologically, troxerutin administration was associated with lower pyramidal cells in the hippocampus, with disorganized layers, karyopyknosis, reduced endochylema and cavitation comparted to the diabetic groups. Gui and colleagues investigated the impact of troxerutin on the CCI-induced neuropathic pain [62]. The results of this study demonstrated that troxerutin significantly reversed mechanical allodynia and thermal hyperalgesia. Furthermore, troxerutin suppressed the expression of INF- $\gamma$, IL- $1 \beta$, TNF- $\alpha$ and activation of NF-kB (p65) as well as prevented microglial activation. Mechanically, it was found that these protective effects of troxerutin are mediated by AMPK activation and upregulation of SIRT1.

\section{Anti-diabetic effect}

The estimates indicate the enhanced incidence rate of diabetes mellitus around the world which has resulted in designing a number of pharmacological options for its treatment and prevention [63-66]. Troxerutin is one of these pharmacological options which has shown great anti-diabetic activity. In a study, the impact of troxerutin on the male fertility in type I diabetes (T1D) rats was evaluated [67]. The results of this study showed that troxerutin effectively decreases blood sugar and also enhances testosterone and glutathione peroxide (GPX), but it has no impact on the insulin, FSH, LH, malondialdehyde (MDA), total antioxidant capacity (TAC) and superoxide dismustase (SOD). Besides, it was found that troxerutin is able to diminish diabetes-mediated testicular structural damage and promote sperm numbers, motility and viability. In a study, Zhang and coworkers evaluated the protective effect of troxerutin on the diabetic cognitive dysfunction [68]. The results of this study showed that rats exposed to troxerutin have higher learning and memory levels. Also, it was found that troxerutin increases SOD activity and diminishes MDA content through nuclear factor
E2-related factor 2 (Nrf2) upregulation. In a study, the protective effect of troxerutin against diabetic cardiomyopathy was assessed [69]. Troxerutin administration was associated with reduction in heart rate, blood pressure, blood glucose and plasma triglyceride levels, ROS concentrations and NF-kB protein expression. Besides, troxerutin prevented the phosphorylation of Akt, insulin receptor substrate 1 (IRS1) and c-Jun N-terminal kinase, demonstrating the potential of troxerutin in protection against diabetic cardiomyopathy. Yavari and colleagues assessed the effect of troxerutin on the inflammatory NF-kB pathway in hippocampus of diabetic rats [70]. It was found that troxerutin effectively reduces hippocampal microRNA levels of NF-kB, interleukin-1 receptor-associated kinase-1 (IRAK1) and tumor necrosis factor receptor-associated factor 6 (TRAF6) via upregulation of miR-146a and miR-155.

\section{Protective effect against ischemic/reperfusion}

Ischemic/reperfusion (I/R) plays a significant role in induction of cell and organ dysfunction and after reperfusion, oxidative stress and inflammatory response result in deterioration of condition. So, finding compounds which prevent $\mathrm{I} / \mathrm{R}$ injury is of interest and troxerutin has shown promising profile in this field. In a study, the inhibitory effect of troxerutin on the myocardial cell apoptosis following myocardial I/R injury was evaluated [71]. The in vitro (cardiomyoctes) and in vivo (rat) demonstrated that troxerutin administration exerts antiapoptotic effect through miR-146a-5p downregulation. Kheirollahi and colleagues examined the protective impact of troxerutin on I/R-induced damages in rat's disease [72]. The results of this study demonstrated potential protective effect of troxerutin on $\mathrm{I} / \mathrm{R}$ injury, so that troxerutin administration significantly increased Johnson score's, sperm count and levels of LH and FSH, and simultaneously, exerted anti-apoptotic effect. In another study, the impact of TCHI on neurovascular units exposed to oxygen-glucose deprivation and reoxygenation was evaluated [73]. It was found that TCHI increases the expression level of GAP-43, Claudin-5 and AQP-4 and attenuates abnormalities of unltrastructure of neurons and rat brain microvascular endothelial cells (rBMECs). Besides, TCHI effectively decreased the levels of inflammatory cytokines (IL-1 $\beta$, IL- 6 and TNF- $\alpha$ ) and cell adhesion molecules (VCAM-1 and ICAM-1) as well as proapoptotic factors (Bax, p53 and caspase-3), showing the efficacy of troxerutin in management of I/R injuries. Najafi and colleagues examined the effect of troxerutin on the I/R in- 
jury of diabetic myocardium [74]. It was shown that treatment with troxerutin decreases the number of premature ventricular (PCV) complexes and duration and incidence of ventricular fibrillation, and also diminishes the duration and incidence of most arrhythmias. Also, it was found that troxerutin administration is associated with decreased level of inflammatory cytokines such as TNF- $\alpha$ and IL-1 $\beta$. Shu and colleagues examined the protective effect of troxerutin against myocardial I/R injury [75]. It was shown that troxerutin administration effectively decreases myocardial infarct size, promotes cardiac function and reduces the levels of creatinine kinase (CK), aspartate aminotransferase (AST) and lactate dehydrogenase (LDH) in the I/ $\mathrm{R}$ injury rat model. Also, troxerutin diminishes the serum and mRNA levels of TNF- $\alpha$ and IL-10 as well as some apoptotic markers (Bax and caspase 3). It was found that these protective effects of troxerutin are mediated by increased phosphorylation of Akt and PI3K inhibition. Badalzadeh and colleagues examined the protective effect of troxerutin after myocardial injury [76]. Pretreatment with troxerutin was associated with reduced level of inflammatory cytokines such as TNF- $\alpha$ and IL-1 $\beta$ and decreased activity of intercellular adhesion molecule-1 (ICAM1). The results of posttreatment with troxerutin were similar to pretreatment results, showing the protective effect of troxerutin against myocardial I/R injury through inhibition of cell-cell interaction and release of inflammatory mediators.

\section{Performance promoting effect}

Zamanian and colleagues examined the antifatigue effect of troxerutin in trained male rats [77]. Troxerutin administration remarkably increased exhaustion swimming time, glucose level and SOD activity, while it decreased creatinine kinase activity, alkaline phosphatase and LDH activities and blood urea nitrogen as well as MMP-9 levels.

\section{Protection against radiation}

As a promising procedure in treatment of tumors, radiotherapy has harmful effects on normal cells. Troxerutin has shown promising profile in inhibiting the adverse effects of irradiation. In a study, $\mathrm{Xu}$ and colleagues examined the effect of troxerutin on radiation-induced PTEN activation [78]. It was found that troxerutin inhibits the apoptosis stimulation by irradiation in thymocytes. Mechanically, troxerutin stimulated the activation of Akt through PTEN inhibition, consequently resulting in JNK prevention and protection of cells.

\section{Cardioprotective effect}

In a study, the effect of troxerutin on the myocardial damage in mice fed fat and fructose-rich diet was evaluated [79]. It was shown that this kind of diet reduces the levels of antioxidant and increases ROS generation, lipid peroxidation and oxidatively modified adducts of 6-OH6, 4-HNE and 3-NT. Also, high fat or fructose diet (HFFD) increased $\mathrm{Ca}^{2+}$ levels and decreased calcium transportes and cardiolipin content. Besides, alterations in mitochondrial structure were observed and cytochrome C release, increase in proapoptotic proteins (APAF-1, Bax, caspase-9 and -3) and reduction in antiapoptotic protein (Bcl-2) demonstrated apoptosis induction. It was found that troxerutin administration alleviates cardiac apoptosis and exerts protective role through its antioxidant effect as well as promoting mitochondrial fusion. In another study, the ameliorative impact of troxerutin on the diet-induced oxidative stress in mice was examined [80]. It was shown that troxerutin administration inhibits oxidative stress and promotes the oxidative capacity and biogenesis and recovers fission/fusion imbalance in the cardiac mitochondria of HFFD-fed mice. Mokhtari and coworkers investigated the impact of troxerutin on reperfusion injury of diabetic myocardium [81]. The results of this study demonstrated that troxerutin administration remarkably diminishes cardiac troponin I (cTnI) levels and apoptotic index via GSK-3 $\beta$ inhibition.

\section{Hepatoprotective effect}

In a study, the effect of troxerutin on the increased hepatic gluconeogenesis in HFD-treated mice was examined [82]. The results of this study indicated the promotion of obesity and related metabolic parameters as well as liver injuries by troxerutin administration. Also, increased level of hepatic gluconeogenesis and upregulation of NF-kB p65 transcriptional activation as well as release of inflammatory cytokines were suppressed following troxerutin administration. It was found that these protective effects of troxerutin are mediated through downregulation of Nucleotide oligomerization domain (NOD) and inhibition of interaction between NOD1/2 with interacting protein-2 (RIP2) through reduction of oxidative stress-mediated endoplasmic reticulum (ER) stress. 


\section{CONCLUSION AND REMARKS}

Flavonoids comprise a wide range of naturally occurring compounds and troxerutin is a bioflavonoid exclusively found in tea, coffee, cereal grains and other fruits and vegetables. Over the past years, much attention has been made towards the troxerutin which is due to its favorable biological activities such as antioxidant, anti-inflammatory, performance promoting effect, protection against I/R, hepatoprotective, neuroprotective, renoprotective and protection against radiation which were discussed in the present review. Mechanically, it was found that antioxidant activity of troxerutin is mainly mediated through inhibition of NOX2 and stimulation of Nrf2 signaling pathway, resulting in reduction in ROS production and improvement in antioxidant capacity. Also, it was found that in order to prevent renal injury from inflammatory responses, troxerutin suppresses the expression of OX-2, iNOS and NF-kB via inhibition of NLRP3, CXCR4 and TXNIP. On the other hand, in order to inhibit the production of inflammatory cytokines, troxerutin is able to stimulate the expression of PCNA. Furthermore, antitumor activity of troxerutin mainly depends on upregulation of HO- 1 and Nrf2 and downregulation of LKKB, NF-kB and STAT3, leading to the inhibition of inflammatory responses, proliferation of tumor cells, and also induction of apoptosis and consequently, suppresses tumor cells. Besides, troxerutin has demonstrated great neuroprotective impact and it is necessary to manifest the molecular signaling pathways of its neuroprotective effect. In order to exert neuroprotective effect, troxerutin stimulates the activation of AMPK, SIRT1, ER $\beta$ and PI3K, resulting in reduction in inflammatory cytokines, MDA and ROS levels, GFAP and DNA fragmentation. However, signaling pathways are like a puzzle and more studies are needed to clarify these pathways in detail.

\section{CONFLICT OF INTEREST}

The authors declare no conflict of interest.

\section{ORCID}

Zahra Ahmadi, https://orcid.org/0000-0002-7605-9303

Reza Mohammadinejad, https://orcid.org/0000-0002-5806-1135

Sahar Roomiani, https://orcid.org/0000-0002-3112-9179

Milad Ashrafizadeh, https://orcid.org/0000-0001-6605-822X

\section{REFERENCES}

1. Ghosh D, Dey SK, Saha C. Antagonistic effects of black tea against gamma radiation-induced oxidative damage to normal lymphocytes in comparison with cancerous K562 cells. Radiat Environ Biophys. 2014;53(4):695-704.

2. Jose J, Sudhakaran S, Sumesh Kumar TM, Jayaraman S, Jayadevi Variyar E. A comparative evaluation of anticancer activities of flavonoids isolated from Mimosa pudica, Aloe vera and Phyllanthus niruri against human breast carcinoma cell line (MCF-7) using MTT assay. Int J Pharm Pharm Sci. 2014;6(2):319-22.

3. Erdman JW Jr, Balentine D, Arab L, Beecher G, Dwyer JT, Folts J, et al. Flavonoids and heart health: proceedings of the ILSI North America Flavonoids Workshop, May 31-June 1, 2005, Washington, DC. J Nutr. 2007;137(3 Suppl 1):718S-37S

4. Mahomoodally MF, Gurib-Fakim A, Subratty AH. Antimicrobial activities and phytochemical profiles of endemic medicinal plants of Mauritius. Pharm Biol. 2005;43(3):237-42.

5. Scalbert A, Williamson G. Dietary intake and bioavailability of polyphenols. J Nutr. 2000;130(8S Suppl):2073S-85S.

6. Williamson G, Clifford MN. Colonic metabolites of berry polyphenols: the missing link to biological activity? Br J Nutr. 2010; 104 Suppl 3:S48-66.

7. Badalzadeh R, Layeghzadeh N, Alihemmati A, Mohammadi M. Beneficial effect of troxerutin on diabetes-induced vascular damages in rat aorta: histopathological alterations and antioxidation mechanism. Int J Endocrinol Metab. 2015;13(2):e25969.

8. Cha HJ, Lee KS, Lee GT, Lee KK, Hong JT, Lee SN, et al. Altered miRNA expression profiles are involved in the protective effects of troxerutin against ultraviolet $\mathrm{B}$ radiation in normal human dermal fibroblasts. Int J Mol Med. 2014;33(4):957-63.

9. Geetha R, Radika MK, Priyadarshini E, Bhavani K, Anuradha CV. Troxerutin reverses fibrotic changes in the myocardium of high-fat high-fructose diet-fed mice. Mol Cell Biochem. 2015; 407(1-2):263-79.

10. Lee KS, Cha HJ, Lee GT, Lee KK, Hong JT, Ahn KJ, et al. Troxerutin induces protective effects against ultraviolet $\mathrm{B}$ radiation through the alteration of microRNA expression in human HaCaT keratinocyte cells. Int J Mol Med. 2014;33(4):934-42.

11. Sampath S, Karundevi B. Effect of troxerutin on insulin signaling molecules in the gastrocnemius muscle of high fat and sucrose-induced type- 2 diabetic adult male rat. Mol Cell Biochem. 2014;395(1-2):11-27

12. Vinothkumar R, Vinoth Kumar R, Sudha M, Viswanathan P, Balasubramanian T, Nalini N. Modulatory effect of troxerutin on biotransforming enzymes and preneoplasic lesions induced by 1,2-dimethylhydrazine in rat colon carcinogenesis. Exp Mol Pathol. 2014;96(1):15-26. 
13. Zhang ZF, Fan SH, Zheng YL, Lu J, Wu DM, Shan Q, et al. Troxerutin improves hepatic lipid homeostasis by restoring $\mathrm{NAD}(+)$ depletion-mediated dysfunction of lipin 1 signaling in high-fat diet-treated mice. Biochem Pharmacol. 2014;91(1):74-86.

14. Zhang ZF, Shan Q, Zhuang J, Zhang YQ, Wang X, Fan SH, et al. Troxerutin inhibits 2,2',4,4'-tetrabromodiphenyl ether (BDE-47)-induced hepatocyte apoptosis by restoring proteasome function. Toxicol Lett. 2015;233(3):246-57.

15. Zhang ZF, Zhang YQ, Fan SH, Zhuang J, Zheng YL, Lu J, et al. Troxerutin protects against 2,2',4,4'-tetrabromodiphenyl ether (BDE-47)-induced liver inflammation by attenuating oxidative stress-mediated $\mathrm{NAD}^{+}$-depletion. J Hazard Mater. 2015;283:98109.

16. Mintz KJ, Mercado G, Zhou Y, Ji Y, Hettiarachchi SD, Liyanage PY, et al. Tryptophan carbon dots and their ability to cross the blood-brain barrier. Colloids Surf B Biointerfaces. 2019;176:48893.

17. Yaribeygi H, Atkin SL, Sahebkar A. Interleukin-18 and diabetic nephropathy: a review. J Cell Physiol. 2019;234(5):5674-82.

18. Yaribeygi H, Butler AE, Atkin SL, Katsiki N, Sahebkar A. Sodium-glucose cotransporter 2 inhibitors and inflammation in chronic kidney disease: possible molecular pathways. J Cell Physiol. 2018;234(1):223-30.

19. Yaribeygi H, Katsiki N, Butler AE, Atkin SL, Sahebkar A. A response to "In response to 'Sodium-glucose cotransporter 2 inhibitors and inflammation in chronic kidney disease: possible molecular pathways"'. J Cell Physiol. 2019;234(7):9908-9.

20. Yaribeygi H, Simental-Mendía LE, Butler AE, Sahebkar A. Protective effects of plant-derived natural products on renal complications. J Cell Physiol. 2019;234(8):12161-72.

21. Salama SA, Arab HH, Maghrabi IA. Troxerutin down-regulates KIM-1, modulates p38 MAPK signaling, and enhances renal regenerative capacity in a rat model of gentamycin-induced acute kidney injury. Food Funct. 2018;9(12):6632-42.

22. Yang X, Xu W, Huang K, Zhang B, Wang H, Zhang X, et al. Precision toxicology shows that troxerutin alleviates ochratoxin Ainduced renal lipotoxicity. FASEB J. 2019;33(2):2212-27.

23. Shan Q, Zheng GH, Han XR, Wen X, Wang S, Li MQ, et al. Troxerutin protects kidney tissue against BDE-47-induced inflammatory damage through CXCR4-TXNIP/NLRP3 signaling. Oxid Med Cell Longev. 2018;2018:9865495.

24. Elangovan P, Ramakrishnan R, Amudha K, Jalaludeen AM, Sagaran GK, Babu FR, et al. Beneficial protective effect of troxerutin on Nickel-induced renal dysfunction in Wistar Rats. J Environ Pathol Toxicol Oncol. 2018;37(1):1-14.

25. Dehnamaki F, Karimi A, Pilevarian AA, Fatemi I, Hakimizadeh E, Kaeidi A, et al. Treatment with troxerutin protects against cisplatin-induced kidney injury in mice. Acta Chir Belg.

\section{9;119(1):31-7.}

26. Shan Q, Zhuang J, Zheng G, Zhang Z, Zhang Y, Lu J, et al. Troxerutin reduces kidney damage against BDE-47-induced apoptosis via inhibiting NOX2 activity and increasing Nrf2 activity. Oxid Med Cell Longev. 2017;2017:6034692.

27. Ambati RR, Phang SM, Ravi S, Aswathanarayana RG. Astaxanthin: sources, extraction, stability, biological activities and its commercial applications--a review. Mar Drugs. 2014;12(1):12852.

28. Edge R, McGarvey DJ, Truscott TG. The carotenoids as antioxidants--a review. J Photochem Photobiol B. 1997;41(3):189200.

29. Heidari Khoei H, Fakhri S, Parvardeh S, Shams Mofarahe Z, Ghasemnejad-Berenji H, Nazarian H, et al. Testicular toxicity and reproductive performance of streptozotocin-induced diabetic male rats: the ameliorating role of silymarin as an antioxidant. Toxin Rev. 2019;38(3):223-33.

30. Najafi H, Changizi Ashtiyani S, Madani SH, Fakhri S, Mohamadi Yarijani Z, Hazem M. Therapeutic effects of curcumin on renal tissue damages induced by ischemia reperfusion in rat. Koomesh. 2014;16(2):273-81.

31. Najafi H, Changizi Ashtiyani S, Sayedzadeh SA, Mohamadi Yarijani Z, Fakhri S. Therapeutic effects of curcumin on the functional disturbances and oxidative stress induced by renal ischemia/reperfusion in rats. Avicenna J Phytomed. 2015;5(6):57686.

32. Valko M, Leibfritz D, Moncol J, Cronin MT, Mazur M, Telser J. Free radicals and antioxidants in normal physiological functions and human disease. Int J Biochem Cell Biol. 2007;39(1):44-84.

33. Wu H, Niu H, Shao A, Wu C, Dixon BJ, Zhang J, et al. Astaxanthin as a potential neuroprotective agent for neurological diseases. Mar Drugs. 2015;13(9):5750-66.

34. Raja B, Saranya D, Prabhu R. Role of flavonoid troxerutin on blood pressure, oxidative stress and regulation of lipid metabolism. Front Biosci (Elite Ed). 2019;11:121-9.

35. Xin X, Zhang M, Li X, Lai F, Zhao G. Biocatalytic synthesis of acylated derivatives of troxerutin: their bioavailability and antioxidant properties in vitro. Microb Cell Fact. 2018;17(1):130.

36. Elangovan P, Jalaludeen AM, Ramakrishnan R, Pari L. Protective effect of troxerutin on Nickel-induced testicular toxicity in Wistar Rats. J Environ Pathol Toxicol Oncol. 2016;35(2):133-46.

37. Panat NA, Maurya DK, Ghaskadbi SS, Sandur SK. Troxerutin, a plant flavonoid, protects cells against oxidative stress-induced cell death through radical scavenging mechanism. Food Chem. 2016;194:32-45.

38. Bahreyni A, Ghorbani E, Fuji H, Ryzhikov M, Khazaei M, Erfani $\mathrm{M}$, et al. Therapeutic potency of oncolytic virotherapy-induced cancer stem cells targeting in brain tumors, current status, and 
perspectives. J Cell Biochem. 2019;120(3):2766-73.

39. Bahreyni A, Rezaei M, Bahrami A, Khazaei M, Fiuji H, Ryzhikov M, et al. Diagnostic, prognostic, and therapeutic potency of microRNA 21 in the pathogenesis of colon cancer, current status and prospective. J Cell Physiol. 2019;234(6):8075-81.

40. Barati Bagherabad M, Afzaljavan F, ShahidSales S, Hassanian SM, Avan A. Targeted therapies in pancreatic cancer: promises and failures. J Cell Biochem. 2019;120(3):2726-41.

41. Ghobadi N, Mehramiz M, ShahidSales S, Rezaei Brojerdi A, Anvari $\mathrm{K}$, Khazaei $\mathrm{M}$, et al. A genetic variant in $\mathrm{CDKN} 2 \mathrm{~A} / 2 \mathrm{~B}$ locus was associated with poor prognosis in patients with esophageal squamous cell carcinoma. J Cell Physiol. 2019;234(4):5070-6.

42. Mahdavi M, Nassiri M, Kooshyar MM, Vakili-Azghandi M, Avan A, Sandry R, et al. Hereditary breast cancer; genetic penetrance and current status with BRCA. J Cell Physiol. 2019; 234(5):5741-50.

43. Marjaneh RM, Khazaei M, Ferns GA, Avan A, Aghaee-Bakhtiari $\mathrm{SH}$. The role of microRNAs in 5-FU resistance of colorectal cancer: possible mechanisms. J Cell Physiol. 2019;234(3):2306-16.

44. Moradi-Marjaneh R, Hassanian SM, Hasanzadeh M, Rezayi M, Maftouh M, Mehramiz M, et al. Therapeutic potential of tolllike receptors in treatment of gynecological cancers. IUBMB Life. 2019;71(5):549-64.

45. Parizadeh SM, Jafarzadeh-Esfehani R, Ghandehari M, Goldani F, Parizadeh SMR, Hassanian SM, et al. MicroRNAs as potential diagnostic and prognostic biomarkers in hepatocellular carcinoma. Curr Drug Targets. 2019;20(11):1129-40.

46. Parizadeh SM, Jafarzadeh-Esfehani R, Hassanian SM, Parizadeh SMR, Vojdani S, Ghandehari M, et al. Targeting cancer stem cells as therapeutic approach in the treatment of colorectal cancer. Int J Biochem Cell Biol. 2019;110:75-83.

47. Taghizadeh Kermani A, Hosseini S, Fanipakdel A, Joudi Mashhad M, Akhavan Rezayat K, Zardadi M, et al. A randomized clinical trial on the antitumoral effects of low molecular weight heparin in the treatment of esophageal cancer. J Cell Physiol. 2019;234(4):4191-9.

48. Thomas NS, George K, Selvam AAA. Anticancer mechanism of troxerutin via targeting $\mathrm{Nrf} 2$ and $\mathrm{NF}-\kappa \mathrm{B}$ signalling pathways in hepatocarcinoma cell line. Toxicol In Vitro. 2019;54:317-29.

49. Thomas NS, George K, Selvam AAA. Troxerutin subdues hepatic tumorigenesis via disrupting the MDM2-p53 interaction. Food Funct. 2018;9:5336-49.

50. Subastri A, Suyavaran A, Preedia Babu E, Nithyananthan S, Barathidasan R, Thirunavukkarasu C. Troxerutin with copper generates oxidative stress in cancer cells: its possible chemotherapeutic mechanism against hepatocellular carcinoma. J Cell Physiol. 2018;233(3):1775-90.

51. Xu GY, Tang XJ. Troxerutin (TXN) potentiated 5-Fluorouracil
(5-Fu) treatment of human gastric cancer through suppressing STAT3/NF- $\mathrm{B}$ and Bcl-2 signaling pathways. Biomed Pharmacother. 2017;92:95-107.

52. Subastri A, Harikrishna K, Sureshkumar M, Alshammari GM, Aristatile B, Thirunavukkarasu C. Effect of troxerutin on 2-aminoanthracene and DNA interaction and its anti-mutagenic property. Biomed Pharmacother. 2017;88:325-34.

53. Thomas NS, George K, Arivalagan S, Mani V, Siddique AI, Namasivayam N. The in vivo antineoplastic and therapeutic efficacy of troxerutin on rat preneoplastic liver: biochemical, histological and cellular aspects. Eur J Nutr. 2017;56(7):2353-66.

54. Panat NA, Singh BG, Maurya DK, Sandur SK, Ghaskadbi SS. Troxerutin, a natural flavonoid binds to DNA minor groove and enhances cancer cell killing in response to radiation. Chem Biol Interact. 2016;251:34-44.

55. Azarfarin M, Farajdokht F, Babri S, Salehpour F, Taghizadeh M, Mohaddes G. Effects of troxerutin on anxiety- and depressivelike behaviors induced by chronic mild stress in adult male rats. Iran J Basic Med Sci. 2018;21(8):781-6.

56. Diba R, Mohaddes G, Mirzaie Bavil F, Farajdokht F, Bayandor P, Hosseindoost M, et al. Protective effects of troxerutin on maternal high-fat diet-induced impairments of spatial memory and apelin in the male offspring. Iran J Basic Med Sci. 2018;21(7): 682-7.

57. Zhào H, Liu Y, Zeng J, Li D, Huang Y. Troxerutin cerebroprotein hydrolysate injection ameliorates neurovascular injury induced by traumatic brain injury- via endothelial nitric oxide synthase pathway regulation. Int J Neurosci. 2018;128(12):1118-27.

58. Bayandor P, Farajdokht F, Mohaddes G, Diba R, Hosseindoost M, Mehri K, et al. The effect of troxerutin on anxiety- and depressive-like behaviours in the offspring of high-fat diet fed dams. Arch Physiol Biochem. 2019;125(2):156-62.

59. Farajdokht F, Amani M, Mirzaei Bavil F, Alihemmati A, Mohaddes G, Babri S. Troxerutin protects hippocampal neurons against amyloid beta-induced oxidative stress and apoptosis. EXCLI J. 2017;16:1081-9.

60. Baluchnejadmojarad T, Jamali-Raeufy N, Zabihnejad S, Rabiee N, Roghani M. Troxerutin exerts neuroprotection in 6-hydroxydopamine lesion rat model of Parkinson's disease: possible involvement of PI3K/ER $\beta$ signaling. Eur J Pharmacol. 2017;801: $72-8$.

61. Zhang S, Li H, Zhang L, Li J, Wang R, Wang M. Effects of troxerutin on cognitive deficits and glutamate cysteine ligase subunits in the hippocampus of streptozotocin-induced type 1 diabetes mellitus rats. Brain Res. 2017;1657:355-60.

62. Gui Y, Li A, Chen F, Zhou H, Tang Y, Chen L, et al. Involvement of AMPK/SIRT1 pathway in anti-allodynic effect of troxerutin in CCI-induced neuropathic pain. Eur J Pharmacol. 
2015;769:234-41.

63. Yaribeygi H, Atkin SL, Ramezani M, Sahebkar A. A review of the molecular pathways mediating the improvement in diabetes mellitus following caloric restriction. J Cell Physiol. 2019;234(6): 8436-42.

64. Yaribeygi H, Atkin SL, Sahebkar A. Natural compounds with DPP-4 inhibitory effects: implications for the treatment of diabetes. J Cell Biochem. 2019;120(7):10909-13.

65. Yaribeygi H, Atkin SL, Simental-Mendía LE, Barreto GE, Sahebkar A. Anti-inflammatory effects of resolvins in diabetic nephropathy: mechanistic pathways. J Cell Physiol. 2019;234(9): 14873-82.

66. Yaribeygi H, Bo S, Ruscica M, Sahebkar A. Ceramides and diabetes mellitus: an update on the potential molecular relationships. Diabet Med. 2020;37(1):11-9.

67. Zavvari Oskuye Z, Mirzaei Bavil F, Hamidian GR, Mehri K, Qadiri A, Ahmadi M, et al. Troxerutin affects the male fertility in prepubertal type 1 diabetic male rats. Iran J Basic Med Sci. 2019; 22(2):197-205.

68. Zhang W, Wang R, Liu W, Wang X, Li P, Zhang W, et al. Tecontaining carbon dots for fluorescence imaging of superoxide anion in mice during acute strenuous exercise or emotional changes. Chem Sci. 2017;9(3):721-7.

69. Yu Y, Zheng G. Troxerutin protects against diabetic cardiomyopathy through NF- $\kappa \mathrm{B} / \mathrm{AKT} / \mathrm{IRS} 1$ in a rat model of type 2 diabetes. Mol Med Rep. 2017;15(6):3473-8.

70. Yavari R, Badalzadeh R, Alipour MR, Tabatabaei SM. Modulation of hippocampal gene expression of microRNA-146a/ microRNA-155-nuclear factor-kappa B inflammatory signaling by troxerutin in healthy and diabetic rats. Indian J Pharmacol. 2016;48(6):675-80.

71. Shu L, Zhang W, Huang G, Huang C, Zhu X, Su G, et al. Troxerutin attenuates myocardial cell apoptosis following myocardial ischemia-reperfusion injury through inhibition of miR-146a-5p expression. J Cell Physiol. 2019;234(6):9274-82.

72. Kheirollahi A, Abbaszadeh A, Anbari K, Rostami B, Ahangari A, Hasanvand A, et al. Troxerutin protect sperm, seminiferous epithelium and pituitary-gonadal axis from torsion-detorsion injury: an experimental study. Int J Reprod Biomed. 2018;16(5):31522.
73. Zhào H, Liu Y, Zeng J, Li D, Zhang W, Huang Y. Troxerutin and cerebroprotein hydrolysate injection protects neurovascular units from oxygen-glucose deprivation and reoxygenationinduced injury in vitro. Evid Based Complement Alternat Med. 2018;2018:9859672.

74. Najafi M, Noroozi E, Javadi A, Badalzadeh R. Anti-arrhythmogenic and anti-inflammatory effects of troxerutin in ischemia/ reperfusion injury of diabetic myocardium. Biomed Pharmacother. 2018;102:385-91.

75. Shu L, Zhang W, Huang C, Huang G, Su G. Troxerutin protects against myocardial ischemia/reperfusion injury via Pi3k/Akt pathway in rats. Cell Physiol Biochem. 2017;44(5):1939-48.

76. Badalzadeh R, Baradaran B, Alihemmati A, Yousefi B, Abbaszadeh A. Troxerutin preconditioning and ischemic postconditioning modulate inflammatory response after myocardial ischemia/ reperfusion injury in rat model. Inflammation. 2017;40(1):13643.

77. Zamanian M, Hajizadeh MR, Esmaeili Nadimi A, Shamsizadeh A, Allahtavakoli M. Antifatigue effects of troxerutin on exercise endurance capacity, oxidative stress and matrix metalloproteinase-9 levels in trained male rats. Fundam Clin Pharmacol. 2017;31(4):447-55.

78. Xu P, Zhang WB, Cai XH, Qiu PY, Hao MH, Lu DD. Activating AKT to inhibit JNK by troxerutin antagonizes radiation-induced PTEN activation. Eur J Pharmacol. 2017;795:66-74.

79. Geetha R, Sathiya Priya C, Anuradha CV. Troxerutin abrogates mitochondrial oxidative stress and myocardial apoptosis in mice fed calorie-rich diet. Chem Biol Interact. 2017;278:74-83.

80. Rajagopalan G, Chandrasekaran SP, Carani Venkatraman A. Troxerutin attenuates diet-induced oxidative stress, impairment of mitochondrial biogenesis and respiratory chain complexes in mice heart. Clin Exp Pharmacol Physiol. 2017;44(1):103-13.

81. Mokhtari B, Badalzadeh R, Alihemmati A, Mohammadi M. Phosphorylation of GSK-3 $\beta$ and reduction of apoptosis as targets of troxerutin effect on reperfusion injury of diabetic myocardium. Eur J Pharmacol. 2015;765:316-21.

82. Zhang Z, Wang X, Zheng G, Shan Q, Lu J, Fan S, et al. Troxerutin attenuates enhancement of hepatic gluconeogenesis by inhibiting NOD activation-mediated inflammation in high-fat diet-treated mice. Int J Mol Sci. 2017;18(1):31. 